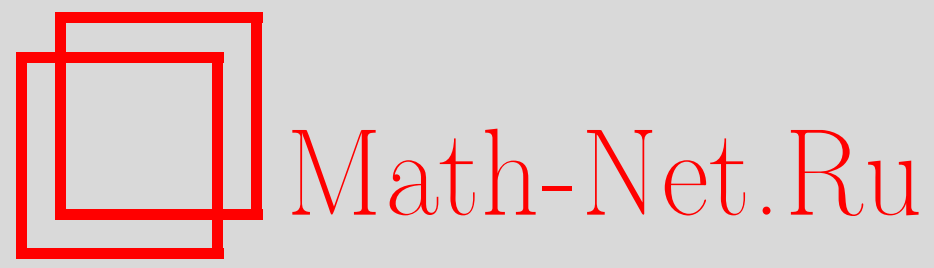

В. В. Григорьева, Ю. В. Шеретов, О точных решениях квазигидродинамической системы, не удовлетворяющих системам Навье-Стокса и Эйлера, Вестник ТвГУ. Серия: Прикладная математика, 2021, выпуск 2, 5-15

DOI: https://doi.org/10.26456/vtpmk611

Использование Общероссийского математического портала Math-Net.Ru подразумевает, что вы прочитали и согласны с пользовательским соглашением

http: //www.mathnet.ru/rus/agreement

Параметры загрузки:

IP : 3.93 .64 .190

26 апреля 2023 г., 15:48:16 


\section{МАТЕМАТИЧЕСКОЕ МОДЕЛИРОВАНИЕ, ЧИСЛЕННЫЕ МЕТОДЫ И КОМПЛЕКСЫ ПРОГРАММ}

УДК $517.95,532.5$

\section{О ТОЧНЫХ РЕШЕНИЯХ КВАЗИГИДРОДИНАМИЧЕСКОЙ СИСТЕМЫ, НЕ УДОВЛЕТВОРЯЮЩИХ СИСТЕМАМ НАВЬЕ-СТОКСА И ЭЙЛЕРА}

Григорьева В.В.*

*Тверской государственный технический университет, г. Тверь

**Тверской государственный университет, г. Тверь

Поступила в редакцию 16.03.2021, после переработки 30.03.2021.

Квазигидродинамическая система была предложена Шеретовым Ю.В. в 1993 году. Известные точные решения этой системы в подавляющем большинстве случаев удовлетворяют либо уравнениям Навье-Стокса, либо уравнениям Эйлера. В настоящей работе описан новый класс точных решений квазигидродинамической системы, которые не удовлетворяют ни уравнениям Навье-Стокса, ни уравнениям Эйлера. Соответствуюшие точные решения системы Навье-Стокса получаются из построенных решений предельным переходом при $c_{s} \rightarrow+\infty$, где $c_{s}-$ скорость звука в жидкости.

Ключевые слова: система Навье-Стокса, система Эйлера, квазигидродинамическая система, точные решения, принцип суперпозиции.

Вестник ТвГУ. Серия: Прикладная математика. 2021. № 2. С. 5-15. https://doi.org/10.26456/vtpmk611

\section{Введение}

Научное направление, связанное с построением точных решений системы Навье-Стокса в динамике вязкой несжимаемой жидкости, представлено во многих монографиях и научных статьях [1-6]. Альтернативная квазигидродинамическая (КГД) модель, имеющая глубокие связи с указанной классической системой, была предложена Шеретовым Ю.В. в 1993 году [7]. Теоретическому обоснованию подхода посвящены монографии $[8,9]$. Найденные точные решения КГД системы в подавляющем большинстве случаев удовлетворяли либо уравнениям Навье Стокса, либо уравнениям Эйлера [8-12]. Единственным исключением было решение, построенное в [8] на с. 106-107 и описывающее течение жидкости в плоском канале с пористыми стенками.

В настоящей работе описан новый класс точных решений квазигидродинамической системы, которые не удовлетворяют ни уравнениям Навье-Стокса, ни

(C) Григорьева В.В., Шеретов Ю.В., 2021 
уравнениям Эйлера. При $c_{s} \rightarrow+\infty$, где $c_{s}-$ скорость звука в жидкости, эти решения стремятся поточечно к соответствующим точным решениям системы НавьеСтокса.

\section{1. Квазигидродинамическая система и система Навье-Стокса}

Квазигидродинамическая система для нестационарных течений слабосжимаемой вязкой жидкости в декартовых координатах имеет вид

$$
\begin{gathered}
\frac{\partial u_{x}}{\partial x}+\frac{\partial u_{y}}{\partial y}+\frac{\partial u_{z}}{\partial z}=\frac{\partial w_{x}}{\partial x}+\frac{\partial w_{y}}{\partial y}+\frac{\partial w_{z}}{\partial z} \\
\frac{\partial u_{x}}{\partial t}+\left(u_{x}-w_{x}\right) \frac{\partial u_{x}}{\partial x}+\left(u_{y}-w_{y}\right) \frac{\partial u_{x}}{\partial y}+\left(u_{z}-w_{z}\right) \frac{\partial u_{x}}{\partial z}+\frac{\partial p}{\partial x}= \\
=\nu\left(\frac{\partial^{2} u_{x}}{\partial x^{2}}+\frac{\partial^{2} u_{x}}{\partial y^{2}}+\frac{\partial^{2} u_{x}}{\partial z^{2}}\right)+\nu \frac{\partial}{\partial x}\left(\frac{\partial u_{x}}{\partial x}+\frac{\partial u_{y}}{\partial y}+\frac{\partial u_{z}}{\partial z}\right)+ \\
+\frac{\partial\left(u_{x} w_{x}\right)}{\partial x}+\frac{\partial\left(u_{y} w_{x}\right)}{\partial y}+\frac{\partial\left(u_{z} w_{x}\right)}{\partial z}, \\
\frac{\partial u_{y}}{\partial t}+\left(u_{x}-w_{x}\right) \frac{\partial u_{y}}{\partial x}+\left(u_{y}-w_{y}\right) \frac{\partial u_{y}}{\partial y}+\left(u_{z}-w_{z}\right) \frac{\partial u_{y}}{\partial z}+\frac{\partial p}{\partial y}= \\
\left.+\frac{\partial^{2} u_{y}}{\partial x^{2}}+\frac{\partial^{2} u_{y}}{\partial y^{2}}+\frac{\partial^{2} u_{y}}{\partial z^{2}}\right)+\nu \frac{\partial}{\partial y}\left(\frac{\partial u_{x}}{\partial x}+\frac{\partial u_{y}}{\partial y}+\frac{\partial u_{z}}{\partial z}\right)+ \\
+\frac{\partial\left(u_{x} w_{y}\right)}{\partial x}+\frac{\partial\left(u_{y} w_{y}\right)}{\partial y}+\frac{\partial\left(u_{z} w_{y}\right)}{\partial z}, \\
\frac{\partial u_{z}}{\partial t}+\left(u_{x}-w_{x}\right) \frac{\partial u_{z}}{\partial x}+\left(u_{y}-w_{y}\right) \frac{\partial u_{z}}{\partial y}+\left(u_{z}-w_{z}\right) \frac{\partial u_{z}}{\partial z}+\frac{\partial p}{\partial z}= \\
=\nu\left(\frac{\partial^{2} u_{z}}{\partial x^{2}}+\frac{\partial^{2} u_{z}}{\partial y^{2}}+\frac{\partial^{2} u_{z}}{\partial z^{2}}\right)+\nu \frac{\partial\left(u_{z} w_{z}\right)}{\partial z}\left(\frac{\partial u_{x}}{\partial x}+\frac{\partial u_{y}}{\partial y}+\frac{\partial u_{z}}{\partial z}\right)+ \\
=
\end{gathered}
$$

Здесь

$$
\begin{aligned}
& w_{x}=\tau\left(u_{x} \frac{\partial u_{x}}{\partial x}+u_{y} \frac{\partial u_{x}}{\partial y}+u_{z} \frac{\partial u_{x}}{\partial z}+\frac{\partial p}{\partial x}\right), \\
& w_{y}=\tau\left(u_{x} \frac{\partial u_{y}}{\partial x}+u_{y} \frac{\partial u_{y}}{\partial y}+u_{z} \frac{\partial u_{y}}{\partial z}+\frac{\partial p}{\partial y}\right), \\
& w_{z}=\tau\left(u_{x} \frac{\partial u_{z}}{\partial x}+u_{y} \frac{\partial u_{z}}{\partial y}+u_{z} \frac{\partial u_{z}}{\partial z}+\frac{\partial p}{\partial z}\right) .
\end{aligned}
$$

Влияние внешних сил не учитывается. Символом $\nu$ обозначен коэффициент кинематической вязкости жидкости. Характерное время релаксации $\tau$ вычисляется по формуле

$$
\tau=\frac{\nu}{c_{s}^{2}}
$$


где $c_{s}$ - скорость звука в жидкости. Параметры $\nu$ и $\tau$ являются положительными константами. Постоянная средняя плотность жидкости $\rho$ положена равной единице. Система (1.1) - (1.4) замкнута относительно неизвестных функций - компонент вектора скорости $u_{x}=u_{x}(x, y, z, t), u_{y}=u_{y}(x, y, z, t), u_{z}=u_{z}(x, y, z, t)$ и давления $p=p(x, y, z, t)$.

Пренебрегая в (1.1) -(1.4) членами, содержащими $\tau$, получим классическую систему Навье-Стокса в динамике вязкой несжимаемой жидкости:

$$
\begin{gathered}
\frac{\partial u_{x}}{\partial x}+\frac{\partial u_{y}}{\partial y}+\frac{\partial u_{z}}{\partial z}=0 \\
\frac{\partial u_{x}}{\partial t}+u_{x} \frac{\partial u_{x}}{\partial x}+u_{y} \frac{\partial u_{x}}{\partial y}+u_{z} \frac{\partial u_{x}}{\partial z}+\frac{\partial p}{\partial x}=\nu\left(\frac{\partial^{2} u_{x}}{\partial x^{2}}+\frac{\partial^{2} u_{x}}{\partial y^{2}}+\frac{\partial^{2} u_{x}}{\partial z^{2}}\right) \\
\frac{\partial u_{y}}{\partial t}+u_{x} \frac{\partial u_{y}}{\partial x}+u_{y} \frac{\partial u_{y}}{\partial y}+u_{z} \frac{\partial u_{y}}{\partial z}+\frac{\partial p}{\partial y}=\nu\left(\frac{\partial^{2} u_{y}}{\partial x^{2}}+\frac{\partial^{2} u_{y}}{\partial y^{2}}+\frac{\partial^{2} u_{y}}{\partial z^{2}}\right) \\
\frac{\partial u_{z}}{\partial t}+u_{x} \frac{\partial u_{z}}{\partial x}+u_{y} \frac{\partial u_{z}}{\partial y}+u_{z} \frac{\partial u_{z}}{\partial z}+\frac{\partial p}{\partial z}=\nu\left(\frac{\partial^{2} u_{z}}{\partial x^{2}}+\frac{\partial^{2} u_{z}}{\partial y^{2}}+\frac{\partial^{2} u_{z}}{\partial z^{2}}\right)
\end{gathered}
$$

\section{2. Частные решения квазигидродинамической системы специального} вида

Будем искать решения системы (1.1) - (1.4) в виде

$$
u_{x}=U, \quad u_{y}=V, \quad u_{z}=\varphi(x, y, t), \quad p=p_{0} .
$$

Здесь $U, V$ и $p_{0}$ - заданные положительные константы. Подстановка (2.1) в (1.5) - (1.7) дает

$$
w_{x}=0, \quad w_{y}=0, \quad w_{z}=\tau\left(U \frac{\partial \varphi}{\partial x}+V \frac{\partial \varphi}{\partial y}\right) .
$$

Для зависимостей (2.1) уравнения (1.1) - (1.3) удовлетворяются тождественно. Уравнение (1.4) запишется следующим образом:

$$
\begin{gathered}
\frac{\partial \varphi}{\partial t}+U \frac{\partial \varphi}{\partial x}+V \frac{\partial \varphi}{\partial y}=\nu\left(\frac{\partial^{2} \varphi}{\partial x^{2}}+\frac{\partial^{2} \varphi}{\partial y^{2}}\right)+ \\
+\tau\left(U^{2} \frac{\partial^{2} \varphi}{\partial x^{2}}+2 U V \frac{\partial^{2} \varphi}{\partial x \partial y}+V^{2} \frac{\partial^{2} \varphi}{\partial y^{2}}\right)
\end{gathered}
$$

Любое решение $\varphi=\varphi(x, y, t)$ уравнения (2.3) порождает точное решение $\left(u_{x}, u_{y}\right.$, $\left.u_{z}, p\right)$ КГД системы (1.1) - (1.4).

Займемся построением частных решений уравнения $(2.3)$. Пусть $\varphi=\varphi(x, t)$. Тогда (2.3) примет вид

$$
\frac{\partial \varphi}{\partial t}+U \frac{\partial \varphi}{\partial x}=\left(\nu+\tau U^{2}\right) \frac{\partial^{2} \varphi}{\partial x^{2}}
$$

Принимая во внимание (1.8), представим (2.4) в эквивалентной форме

$$
\frac{\partial \varphi}{\partial t}+U \frac{\partial \varphi}{\partial x}=\nu_{1} \frac{\partial^{2} \varphi}{\partial x^{2}}
$$


Здесь

$$
\nu_{1}=\nu\left(1+\left(\frac{U}{c_{s}}\right)^{2}\right)
$$

Дополним (2.5) начальным условием

$$
\left.\varphi\right|_{t=0}=\varphi_{0}(x), \quad x \in \mathbb{R} .
$$

Здесь $\varphi_{0}(x)=u_{z}-$ заданная функция. Решение одномерного нестационарного уравнения конвекции-диффузии (2.5) будем искать в виде

$$
\varphi=\Phi(\xi, t)
$$

где

$$
\xi=x-U t .
$$

Подставив (2.8) в (2.5), получим классическое линейное уравнение теплопроводности

$$
\frac{\partial \Phi}{\partial t}=\nu_{1} \frac{\partial^{2} \Phi}{\partial \xi^{2}}
$$

Из (2.7) и (2.9) следует, что к (2.10) необходимо присоединить начальное условие

$$
\left.\Phi\right|_{t=0}=\varphi_{0}(\xi), \quad \xi \in \mathbb{R}
$$

Решение задачи Коши (2.10), (2.11) дается [13] формулой Пуассона

$$
\Phi(\xi, t)=\frac{1}{2 \sqrt{\pi \nu_{1} t}} \int_{-\infty}^{+\infty} \varphi_{0}\left(x_{*}\right) \exp \left(-\frac{\left(\xi-x_{*}\right)^{2}}{4 \nu_{1} t}\right) d x_{*}, \quad t>0 .
$$

Следствием (2.8), (2.9), (2.12) является зависимость

$$
\varphi(x, t)=\frac{1}{2 \sqrt{\pi \nu_{1} t}} \int_{-\infty}^{+\infty} \varphi_{0}\left(x_{*}\right) \exp \left(-\frac{\left(x-U t-x_{*}\right)^{2}}{4 \nu_{1} t}\right) d x_{*}, \quad t>0
$$

которая определяет решение задачи Коши (2.5), (2.7).

\section{3. Примеры точных решений. Принцип суперпозиции}

Приведем примеры точных решений квазигидродинамической системы.

Пример 1. Будем искать решение уравнения (2.10) в виде

$$
\Phi(\xi, t)=A \sin \left(\frac{\xi}{H}\right) \exp (-\lambda t) .
$$

Здесь $A$ и $H$ - положительные константы, имеющие размерности $c \mathcal{M} / c$ и $c$ м соответственно. Подставляя (3.1) в (2.10), находим

$$
\lambda=\frac{\nu_{1}}{H^{2}} .
$$


Из (3.1) и (3.2) следует, что

$$
\Phi(\xi, t)=A \sin \left(\frac{\xi}{H}\right) \exp \left(-\frac{\nu_{1} t}{H^{2}}\right) .
$$

Используя $(2.1),(2.8),(2.9)$ и (3.3), выписываем точное решение КГД системы

$$
u_{x}=U, \quad u_{y}=V, \quad u_{z}=A \sin \left(\frac{x-U t}{H}\right) \exp \left(-\frac{\nu_{1} t}{H^{2}}\right), \quad p=p_{0} .
$$

Пример 2. Пусть $B$ и $t_{0}$ - положительные постоянные, имеющие размерности $c \mathcal{M}^{2} / c$ и $c$ соответственно. Функция

$$
\Phi(\xi, t)=\frac{B}{2 \sqrt{\pi \nu_{1}\left(t+t_{0}\right)}} \exp \left(-\frac{\xi^{2}}{4 \nu_{1}\left(t+t_{0}\right)}\right), \quad t>0,
$$

задает [13] точное решение уравнения теплопроводности (2.10). Соответствующее точное решение системы КГД имеет вид

$$
u_{x}=U, \quad u_{y}=V, \quad u_{z}=\frac{B}{2 \sqrt{\pi \nu_{1}\left(t+t_{0}\right)}} \exp \left(-\frac{(x-U t)^{2}}{4 \nu_{1}\left(t+t_{0}\right)}\right), \quad p=p_{0} .
$$

Пример 3. Непосредственной проверкой можно убедиться в том, что набор функций

$$
\begin{gathered}
u_{x}=U, \quad u_{y}=V, \quad u_{z}=A \sin \left(\frac{x-U t}{H}\right) \exp \left(-\frac{\nu_{1} t}{H^{2}}\right)+ \\
+\frac{B}{2 \sqrt{\pi \nu_{1}\left(t+t_{0}\right)}} \exp \left(-\frac{(x-U t)^{2}}{4 \nu_{1}\left(t+t_{0}\right)}\right), \quad p=p_{0},
\end{gathered}
$$

также представляет собой точное решение КГД системы (1.1) - (1.4). При этом составляющая скорости $u_{z}$ является формальной алгебраической суммой (суперпозицией) соответствующих компонент $u_{z}$ из двух предыдущих примеров.

Пусть функции $\varphi_{1}=\varphi_{1}(x, t)$ и $\varphi_{2}=\varphi_{2}(y, t)$ удовлетворяют соответственно уравнениям

и

$$
\frac{\partial \varphi_{1}}{\partial t}+U \frac{\partial \varphi_{1}}{\partial x}=\nu_{1} \frac{\partial^{2} \varphi_{1}}{\partial x^{2}}
$$

$$
\frac{\partial \varphi_{2}}{\partial t}+V \frac{\partial \varphi_{2}}{\partial y}=\nu_{2} \frac{\partial^{2} \varphi_{2}}{\partial y^{2}}
$$

Здесь

$$
\nu_{1}=\nu\left(1+\left(\frac{U}{c_{s}}\right)^{2}\right), \quad \nu_{2}=\nu\left(1+\left(\frac{V}{c_{s}}\right)^{2}\right) .
$$

Непосредственной проверкой можно убедиться в том, что функция

$$
\varphi(x, y, t)=\varphi_{1}(x, t)+\varphi_{2}(y, t)
$$


является решением уравнения (2.3). Принцип суперпозиции (3.10) можно проиллюстрировать на примере.

Пример 4. Набор функций

$$
\begin{gathered}
u_{x}=U, \quad u_{y}=V, \quad u_{z}=A \sin \left(\frac{x-U t}{H}\right) \exp \left(-\frac{\nu_{1} t}{H^{2}}\right)+ \\
+\frac{B}{2 \sqrt{\pi \nu_{2}\left(t+t_{0}\right)}} \exp \left(-\frac{(y-V t)^{2}}{4 \nu_{2}\left(t+t_{0}\right)}\right), \quad p=p_{0},
\end{gathered}
$$

образует точное решение КГД системы (1.1) - (1.4).

Пример 5. Убеждаемся в том, что функция

$$
\varphi=\varphi(x, y, t)=C((x-U t)(y-V t)+2 \tau U V t)
$$

удовлетворяет уравнению (2.3). Здесь положительная константа $C$ имеет размерность $c \mathcal{M}^{-1} c^{-1}$. Соответствуюшее точное решение системы КГД имеет вид

$$
u_{x}=U, \quad u_{y}=V, \quad u_{z}=C((x-U t)(y-V t)+2 \tau U V t), \quad p=p_{0},
$$

Заметим, что все построенные решения квазигидродинамической системы не удовлетворяют ни системе Навье-Стокса, ни системе Эйлера. Однако соответствующие точные решения системы Навье-Стокса можно получить формальным предельным переходом при $c_{s} \rightarrow+\infty$.

\section{Заключение}

Актуальной и непростой является задача о нахождении новых классов точных решений квазигидродинамической системы, не удовлетворяющих классическим уравнениям гидродинамики. Это позволило бы понять, при каких условиях наблюдаются заметные расхождения решений КГД системы и системы НавьеСтокса, и какое из решений лучше соответствует экспериментальным данным. Использованию регуляризованных уравнений гидродинамики для построения новых вычислительных алгоритмов посвящены работы [14-17].

\section{Список литературы}

[1] Лойцянский Л.Г. Механика жидкости и газа. М.: Наука, 1987. 840 с.

[2] Ландау Л.Д., Лифшиц Е.М. Гидродинамика. М.: Наука, 1986. 736 с.

[3] Riley N., Drazin P.G. The Navier-Stokes equations: A classification of flows and exact solutions. Cambridge: Cambridge University Press, 2006. 196 p. 
[4] Шмыглевский Ю.Д. Аналитические исследования динамики газа и жидкости. М.: Эдиториал УРСС, 1999. 232 с.

[5] Пухначев В.В. Симметрии в уравнениях Навье-Стокса // Успехи механики. 2006. № 1. C. $6-76$.

[6] Wang C.Y. Exact solutions of the unsteady Navier-Stokes equations // Applied Mechanics Reviews. 1989. Vol. 42, № 11. Part 2. Pp. S269-S282.

[7] Шеретов Ю.В. О единственности решений одной диссипативной системы гидродинамического типа // Математическое моделирование. 1994. Т. 6, № 10. C. $35-45$.

[8] Шеретов Ю.В. Динамика сплошных сред при пространственно-временном осреднении. М., Ижевск: НИЦ «Регулярная и хаотическая динамика», 2009. $400 \mathrm{c}$.

[9] Шеретов Ю.В. Регуляризованные уравнения гидродинамики. Тверь: Тверской государственный университет, 2016. 222 с.

[10] Шеретов Ю.В. О решениях задачи Коши для квазигидродинамической системы // Вестник ТвГУ. Серия: Прикладная математика. 2020. № 1. С. 84-96. https://doi.org/10.26456/vtpmk557

[11] Шеретов Ю.В. О классах точных решений квазигидродинамической системы // Вестник ТвГУ. Серия: Прикладная математика. 2020. № 2. С. 5-17. https://doi.org/10.26456/vtpmk592

[12] Шеретов Ю.В. О построении точных решений двумерной квазигидродинамической системы // Вестник ТвГУ. Серия: Прикладная математика. 2021. № 1. C. 5-20. https://doi.org/10.26456/vtpmk605

[13] Владимиров В.С. Уравнения математической физики. М.: Наука, 1971. 512 с.

[14] Стенина Т.В., Елизарова Т.Г., Крапошин М.В. Регуляризованные уравнения гидродинамики в задаче моделирования дискового насоса и их реализация в рамках программного комплекса OpenFOAM. Препринты ИПМ им. М.В.Келдыша. 2020. 30 c. https://doi.org/10.20948/prepr-2020-66

[15] Стенина Т.В., Елизарова Т.Г., Крапошин М.В. Регуляризованные уравнения гидродинамики в задаче моделирования дискового насоса и их реализация в рамках программного комплекса OpenFOAM. Препринты ИПМ им. M.В.Келдыша. 2020. 30 c. https://doi.org/10.20948/prepr-2020-66

[16] Balashov V.A., Zlotnik A.A. An energy dissipative semi-discrete finitedifference method on staggered meshes for the 3D compressible isothermal Navier-Stokes-Cahn-Hilliard equations // Journal of Computational Dynamics. 2020. Vol. 7, № 2. Pp. 291-312. https://doi.org/10.3934/jcd.2020012

[17] Балашов В.А., Савенков Е.Б. Регуляризованная модель типа фазового поля для описания динамики системы «жидкость-твердое тело». Препринты ИПМ им. М.В.Келдыша. 2020. 29 c. https://doi.org/10.20948/prepr-2020-96 
[18] Balashov V.A., Zlotnik A.A. On a new spatial discretization for a regularized 3D compressible isothermal Navier-Stokes-Cahn-Hilliard system of equations with boundary conditions // Journal of Scientific Computing. 2021. Vol. 86, № 33. Pp. 1-30. https://doi.org/10.1007/s10915-020-01388-6

\section{Образец цитирования}

Григорьева В.В., Шеретов Ю.В. О точных решениях квазигидродинамической системы, не удовлетворяющих системам Навье-Стокса и Эйлера // Вестник ТвГУ. Серия: Прикладная математика. 2021. №2. С. 5-15. https://doi.org/10.26456/vtpmk611

\section{Сведения об авторах}

\section{1. Григорьева Вера Владимировна}

доцент кафедры высшей математики Тверского государственного технического университета.

Россия, 170026, г. Тверь, наб. А. Никитина, д. 22, ТвГТУ.

E-mail: pontida@list.ru

\section{2. Шеретов Юрий Владимирович}

заведующий кафедрой математического анализа Тверского государственного университета.

Россия, 170100, г. Тверь, ул. Желябова, д. З3, ТвГУ.

E-mail: Sheretov.YV@tversu.ru 


\title{
ON EXACT SOLUTIONS OF QUASI-HYDRODYNAMIC SYSTEM THAT DON'T SATISFY THE NAVIER-STOKES AND EULER SYSTEMS
}

\author{
Grigoryeva Vera Vladimirovna \\ Associate Professor at the Department of Higher Mathematics, \\ Tver State Technical University \\ Russia, 170026, Tver, A. Nikitin emb., 22, TvSTU. \\ E-mail: pontida@list.ru \\ Sheretov Yurii Vladimirovich \\ Head of Mathematical Analysis Department, Tver State University \\ Russia, 170100, Tver, Zhelyabov st., 33, TverSU. \\ E-mail: Sheretov. YV@tversu.ru
}

\begin{abstract}
Received 16.03.2021, revised 30.03.2021.
The quasi-hydrodynamic system was proposed by Sheretov Yu.V. in 1993. The known exact solutions of this system in the overwhelming majority of cases satisfy either the Navier-Stokes equations or the Euler equations. This paper describes a new class of exact solutions of quasi-hydrodynamic system that satisfy neither the Navier-Stokes equations, nor the Euler equations. The corresponding exact solutions of the Navier-Stokes system are obtained from the constructed solutions by passing to the limit at $c_{s} \rightarrow+\infty$, where $c_{s}$ is the sonic velocity in the fluid.
\end{abstract}

Keywords: Navier-Stokes system, Euler system, quasi-hydrodynamic system, exact solutions, principle of superposition.

\section{Citation}

Grigoryeva V.V., Sheretov Yu.V., "On exact solutions of quasi-hydrodynamic system that don't satisfy the Navier-Stokes and Euler systems", Vestnik TvGU. Seriya: Prikladnaya Matematika /Herald of Tver State University. Series: Applied Mathematics], 2021, № 2, 5-15(in Russian). https://doi.org/10.26456/vtpmk611

\section{References}

[1] Lojtsyanskij L.G., Mekhanika zhidkosti i gaza [Fluid and Gas Mechanics], Nauka Publ., Moscow, 1987 (in Russian), 840 pp.

[2] Landau L.D., Lifshits E.M., Gidrodinamika [Hydrodynamics], Nauka Publ., Moscow, 1986 (in Russian), 736 pp.

[3] Riley N., Drazin P.G., The Navier-Stokes equations: A classification of flows and exact solutions, Cambridge University Press, Cambridge, 2006, 196 pp.

[4] Shmyglevskij Yu.D., Analiticheskie issledovaniya dinamiki gaza i zhidkosti [Analytical Investigations of Gas and Fluid Dynamics], Editorial URSS Publ., Moscow, 1999 (in Russian), 232 pp. 
[5] Pukhnachev V.V., "Symmetries in the Navier-Stokes equations", Uspekhi mekhaniki [Achievements in Mechanics], 2006, № 1, 6-76 (in Russian).

[6] Wang C.Y., "Exact solutions of the unsteady Navier-Stokes equations", Applied Mechanics Reviews, 42:11, Part 2 (1989), S269-S282.

[7] Sheretov Yu.V., "On uniqueness of the solutions for one dissipative system of hydrodynamic type", Matematicheskoe modelirovanie [Mathematical Modeling], 6:10 (1994), 35-45 (in Russian).

[8] Sheretov Yu.V., Dinamika sploshnykh sred pri prostranstvenno-vremennom osrednenii [Continuum Dynamics under Spatiotemporal Averaging], Regular and Chaotic Dynamics Publ., Moscow, Izhevsk, 2009 (in Russian), 400 pp.

[9] Sheretov Yu.V., Regulyarizovannye uravneniya gidrodinamiki [Regularized Hydrodynamic Equations], Tver State University, Tver, 2016 (in Russian), 222 pp.

[10] Sheretov Yu.V., "On the solutions of Cauchy problem for quasi-hydrodynamic system", Vestnik TvGU. Seriya: Prikladnaya Matematika [Herald of Tver State University. Series: Applied Mathematics], 2020, №1, 84-96 (in Russian), https://doi.org/10.26456/vtpmk557.

[11] Sheretov Yu.V., "On classes of exact solutions of quasi-hydrodynamic system", Vestnik TvGU. Seriya: Prikladnaya Matematika [Herald of Tver State University. Series: Applied Mathematics], 2020, № 2, 5-17 (in Russian), https://doi.org/10.26456/vtpmk592.

[12] Sheretov Yu.V., "On the construction of exact solutions of two-dimensional quasihydrodynamic system", Vestnik TvGU. Seriya: Prikladnaya Matematika [Herald of Tver State University. Series: Applied Mathematics], 2021, № 1, 5-20 (in Russian), https://doi.org/10.26456/vtpmk605.

[13] Vladimirov V.S., Uravneniya Matematicheskoi Fiziki [Equations of Mathematical Physics], Nauka Publ., Moscow, 1971 (in Russian), 512 pp.

[14] Stenina T.V., Elizarova T.G., Kraposhin M.V., Regularized equations for disk pump simulation problems in OpenFOAM implementation, Keldysh Institute of Applied Mathematics Preprints, $2020 \quad$ (in Russian), 30 pp., https://doi.org/10.20948/prepr-2020-66.

[15] Stenina T.V., Elizarova T.G., Kraposhin M.V., Regularized equations for disk pump simulation problems in OpenFOAM implementation, Keldysh Institute of Applied Mathematics Preprints, 2020 (in Russian), 30 pp., https://doi.org/10.20948/prepr-2020-66.

[16] Balashov V.A., Zlotnik A.A., "An energy dissipative semi-discrete finitedifference method on staggered meshes for the 3D compressible isothermal Navier-Stokes-Cahn-Hilliard equations", Journal of Computational Dynamics, 7:2 (2020), 291-312, https://doi.org/10.3934/jcd.2020012. 
[17] Balashov V.A., Savenkov E.B., Regularized phase-field model for description of dynamics of "solid-fluid" system, Keldysh Institute of Applied Mathematics Preprints, 2020 (in Russian), 29 pp., https://doi.org/10.20948/prepr-2020-96.

[18] Balashov V.A., Zlotnik A.A., "On a new spatial discretization for a regularized 3D compressible isothermal Navier-Stokes-Cahn-Hilliard system of equations with boundary conditions", Journal of Scientific Computing, 86:33 (2021), 1-30, https://doi.org/10.1007/s10915-020-01388-6. 